\title{
Soliciting hydrothermal systems: the case of La Soufrière of Guadeloupe (FWI) unrest
}

ROBERT ${ }^{1}$, ROBERTO MORETTI ${ }^{2}$, SEVERINE MOUNE $^{3}$, DAVID JESSOP ${ }^{4}$, MAGALI BONIFACIE ${ }^{5}$ AND CHAGNON GLYNN ${ }^{4}$

${ }^{1}$ Institut de Physique du Globe de Paris

${ }^{2}$ Université de Paris, Institut de Physique du Globe de Paris / Obs. Volcanol. Sismol. Guadeloupe

${ }^{3}$ Université Clermont Auvergne Laboratoire Magmas et Volcans ${ }^{4}$ IPGP

${ }^{5}$ IPGP/CNRS UMR 7154

Presenting Author: moretti@ipgp.fr

We have re-assessed the main physicochemical features of the hydrothermal system of La Soufrière of Guadeloupe (FWI) andesitic volcano. A careful analysis of different techniques adopted historically for gas sampling and analysis by the OVSGIPGP [1] has allowed us to extend the use of our modelling and of gas indicators from both non-condensable and reactive species as back as possible to the last 20 years, also including data from the 1976-77 phreatic eruption. Long-term P-T fluctuations characterize the behaviour of the hydrothermal system in relation to injections of magmatic, deep-sourced fluids into the hydrothermal system. Besides, based on the performance of assessed indicators in relation to the seismic activity showed during this period, we could identify reliable precursors of hydrothermal activity and set possible thresholds for next crises. The main test is made with reference to the 2018 episode of accelerated unrest, which was interpreted as an aborted phreatic eruption [2]. The identified geochemical indicators allow tracking the rapid temperature increase and pressure build-up of the hydrothermal system that may take place in the order of days. The identification of geochemical scenarios associated with the sudden decompression of critical fluids demand a quantum leap in the monitoring strategies which will be briefly discussed.

[1] R. Moretti et al., 2020a, Italian Journal of Geosciences 139 (3), 398-412

[2] R. Moretti et al., 2020b, Journal of Volcanology and Geothermal Research 393, 106769 